\title{
Multifinger Feature Level Fusion Based Fingerprint Identification
}

\author{
Praveen N \\ Research FellowAudio and Image Research \\ Laboratory Department of ElectronicsCochin \\ University of Science and Technology \\ Cochin, Kerala, India.
}

\author{
Tessamma Thomas \\ Professor \\ Department of Electronics \\ Cochin University of Science and Technology \\ Cochin, Kerala, India.
}

\begin{abstract}
Fingerprint based authentication systems are one of the cost-effective biometric authentication techniques employed for personal identification. As the data base population increases, fast identification/recognition algorithms are required with high accuracy. Accuracy can be increased using multimodal evidences collected by multiple biometric traits. In this work, consecutive fingerprint images are taken, global singularities are located using directional field strength and their local orientation vector is formulated with respect to the base line of the finger. Feature level fusion is carried out and a 32 element feature template is obtained. A matching score is formulated for the identification and $100 \%$ accuracy was obtained for a database of 300 persons. The polygonal feature vector helps to reduce the size of the feature database from the present 70-100 minutiae features to just 32 features and also a lower matching threshold can be fixed compared to single finger based identification.
\end{abstract}

Keywords- fingerprint; multimodal biometrics; gradient; orientation field; singularity; matching score.

\section{INTRODUCTION}

Personal authentication based on biometric traits is the most common in the current security access technologies. As the criminal/fraudulent activities are increasing enormously, designing high security identification has always been the main goal in the security business. Biometrics deals with identification of people by their physical and/or behavioral characteristics and, so, inherently requires that the person to be identified is physically present at the point of identification. Fingerprints offer an infallible means of personal identification [1].The large numbers of fingerprint images, which are collected for criminal identification or in business for security purpose, continuously increase the importance of automatic fingerprint identification systems. Most of the automatic fingerprint identification systems can reach around 97\% accuracy with a small database and the accuracy of identification is drops down as the size of database is growing up $[2,3]$. Also, the processing speed of automatic identification systems decreases if it involves a large number of detection features. Hence feature code template size has to be minimized so that identification may be much easier. A fingerprint is characterized by singularities- which are small regions where ridge lines forms the distinctive shapes: loop, delta or whorl (Fig.1). Singularities play a key role in classification of fingerprints $[1,5]$ which sets fingerprints into a specific set.
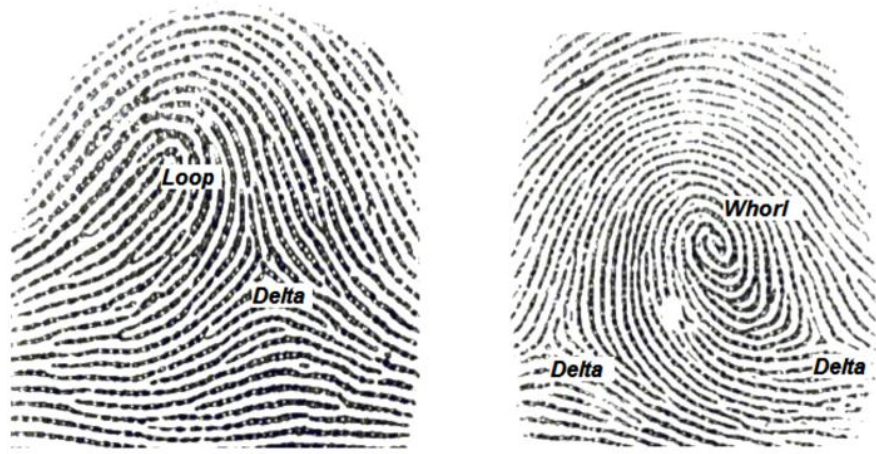

Fig.1. Fingerprint Singularities

Classification eases the searching and in many of the AFIS, classification is the primary procedure adopted [6].

According to Galton-Henry classification scheme [1, 4] there are four common classes of fingerprints: Arch and Tented Arch, Left loop, Right Loop and Whorl (Fig. 2). Cappelli and Maltoni, 2009 [7] studied the spatial distribution of fingerprint singularities and proposed a statistical model for the four most common classes: Arch, Left loop, Right loop and Whorl. The model they proposed gives a clear indication of the fingerprint identity and is used here for identification with a sharp reference position.

Biometric systems that use a single modality are usually affected by problems like noisy sensor data, non-universality and/or lack of distinctiveness of the biometric trait, unacceptable error rates, and spoof attacks [8]. Multibiometric system deals with two or more evidences that are taken from different sources like multiple fingers of the same person,

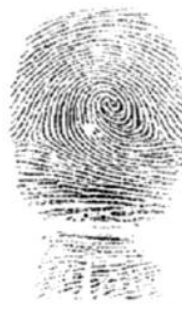

Whorl

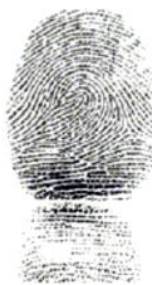

Left Loop

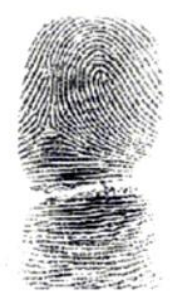

Right Loop

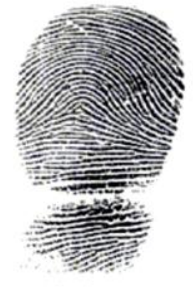

Arch

Fig.2. Fingerprint Types 


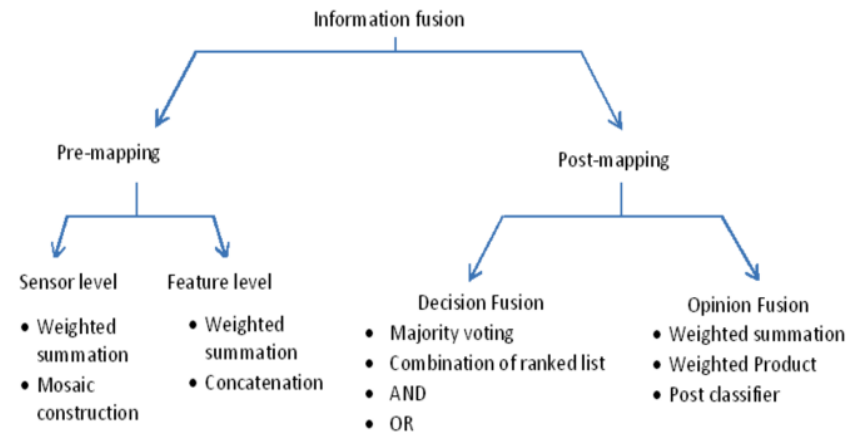

Fig.3. Information fusion

multiple samples of the same instances, multiple sensors for the same biometric, multiple algorithms for representation and matching of multiple traits [9]. Information fusion refers to the consolidating of information or evidences presented by multiple biometric sources $[10,11,12]$.

\section{FUSION IN BIOMETRICS}

Hall and Llinas[13], Ross and Jain[8] have divided information fusion into several categories: sensor level fusion, feature level fusion, score level fusion and decision level fusion. Based on this Sanderson and Paliwal[14] have classified information fusion into pre-mapping fusion, midstmapping fusion and post-mapping fusion [Fig. 3]. Premapping fusion refers to combining information before any use of classifiers or experts. In midst-mapping fusion, information is combined during mapping from sensordata/feature space into opinion/decision space and in post-mapping fusion, information is combined after the decisions of the classifiers have been obtained. Match score fusion based multibiometric algorithm have been developed by Ross and Jain, 2003[8], Frischholz and Dieckmann, 2000[15], Hong and Jain, 1998[16], Biguin et al., 1997[17], Wang et al., 2003[18], Kumar and Zhang, 2003[19]. Fusion at the match score, rank and decision level have been developed and studied extensively. Feature level fusion, however, is a relatively understudied problem [20].

In the present work, feature level fusion of feature vectors is done by concatenating individual feature vectors of consecutive fingers. Fingerprint baseline, which is defined as the line between the Distal and Intermediate Phalangeal join line is taken as the reference line [Fig. 4].This line is detected using correlation technique, singularities are detected using directional field strength and a polygon is formed with

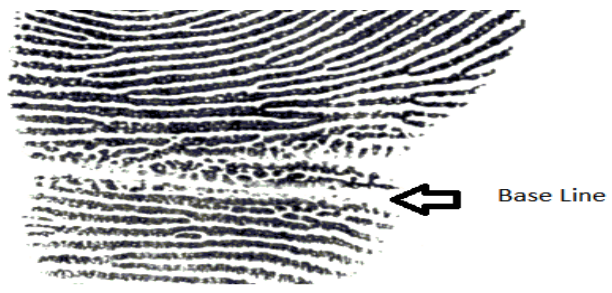

Fig.4. Fingerprint Baseline

singularities and the baseline. For each finger, feature vector is computed as the distance, angle parameters and ridge counts which are concatenated to form the multifinger feature vector. Matching score is formulated to identify the fingerprint. FAR and FRR curves are plotted.

\section{DEFINITION Of THE NOVEL FINGERPRINT STRUCTURE AND FEATURE VECTOR FORMATION}

In this work, fingerprint singularities are identified and a polygon is formed with the baseline [21] [Fig.5]. The polygon thus formed is invariant to rotation. Feature vector describing the polygon is defined as $F=(d, \theta, A, T, r)^{\mathrm{T}}$ where $d$ is the distance metric, $\theta$ is the angle metric, $A$ is the area of the polygon, $T$ is the type of the fingerprint/polygon and $r$ is the ridge counts. The feature vector thus formed is a 16 element vector as:

$$
\left[d_{c c}, d_{c b}, d_{c d r}, d_{d b r}, d_{b b}, d_{d b l}, d_{c d l}, \theta_{c}, \theta_{d r}, \theta_{d l}, \theta_{c c}, A, T, r_{c d}, r_{c b}, r_{d b}\right]^{\mathrm{T}}
$$

where $d_{c c}, d_{c b} . . d_{c d l}$ are the distance measures, $\theta_{c}, \theta_{d r} . . \theta_{c c}$ are the convex angle metrics, $A$ is the Area of the polygon formed and $r_{c d}, r_{c b} \& r_{d b}$ are the ridge counts between core-delta, corebase and delta-base respectively. These are shown in fig.5. Following steps are carried out for constructing the fingerprint polygon:

\section{A. Directional Field Estimation and Strength}

Directional field shows the coarse structure or basic shape of a fingerprint [22] which gives the global information about a fingerprint image. It is defined as the local orientation of the ridge-valley structures.

By computing directional field, singularities can be efficiently located. Several methods have been adopted to estimate directional field. [23], [24], [25]. M. Kass and Witkin, [26] introduced the gradient based method and was adopted by fingerprint researchers $[27,28,29,30]$. This method is $\left[\frac{\partial I(x, y)}{\partial x}\right]$ used in this work.

The gradient $\left[G_{y}(x, y)\right]=\nabla I(x, y)=\left[\frac{\partial I(x, y)}{\partial y}\right] \quad$ vector $\left[G_{x}(x, y) \quad G_{y}(x, y)\right]^{T}$ is defined as:

Where $I(x, y)$ represents the gray-scale image. The directional field is perpendicular to the gradients. Gradients are orientations at pixel-scale whereas directional field describes orientation of ridge-valley structure.

An averaging operation is done on the gradients to obtain the directional field. Gradients cannot be averaged in the local neighborhood as opposite gradients will cancel each other. To solve this problem Kass and Witkin doubled the angle of the gradient vectors before averaging. Doubling makes opposite vectors points in the same 


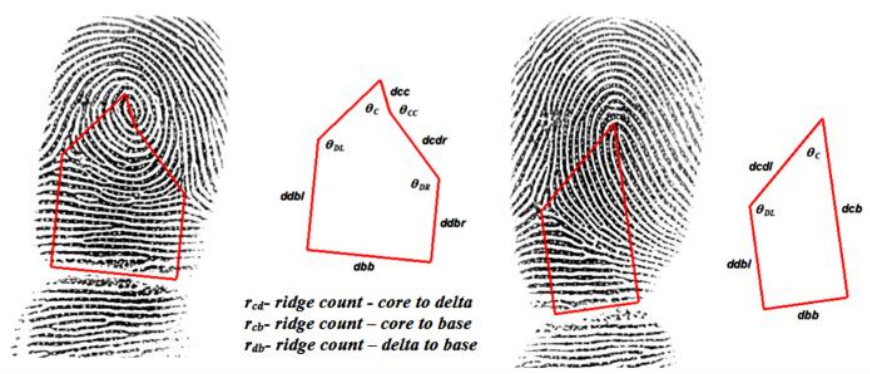

Fig.5. Fingerprint Polygon

direction and will reinforce each other while perpendicular gradients will cancel each other. After averaging, the gradient vectors have to be converted back to their single-angle representation.

The gradient vectors are estimated first in Cartesian coordinate system and is given by $\left[G_{x}, G_{y}\right]$. For the purpose of doubling the angle and squaring the length, the gradient vector is converted to polar system, which is given by

$$
\begin{aligned}
{[\rho \varphi]^{\mathrm{T}} \text { where }-\frac{1}{2} \pi<\phi \leq \frac{1}{2} \pi } \\
{\left[\begin{array}{c}
\rho \\
\varphi
\end{array}\right]=\left[\begin{array}{l}
\sqrt{G_{x}^{2}+G_{y}^{2}} \\
\tan ^{-1} G_{y} / G_{x}
\end{array}\right] }
\end{aligned}
$$

The gradient vector is converted back to its Cartesian as:

$$
\left[\begin{array}{l}
G_{x} \\
G_{y}
\end{array}\right]=\left[\begin{array}{l}
\rho \cos \varphi \\
\rho \sin \varphi
\end{array}\right]
$$

The average squared gradient $\left[\begin{array}{ll}\overline{G_{s, x}} & \overline{G_{s, y}}\end{array}\right]^{T}$ is given by

$$
\left[\begin{array}{c}
\overline{G_{s, x}} \\
\overline{G_{s, y}}
\end{array}\right]=\left[\begin{array}{l}
\sum_{W} G_{x}^{2}-G_{y}^{2} \\
\sum_{W} 2 G_{x} G_{y}
\end{array}\right]=\left[\begin{array}{c}
G_{x x}-G_{y y} \\
2 G_{x y}
\end{array}\right]
$$

where

$$
\begin{aligned}
G_{x x} & =\sum_{W} G_{x}^{2} \\
G_{y y} & =\sum_{W} G_{y}^{2} \\
G_{x y} & =\sum_{W} G_{x} G_{y}
\end{aligned}
$$

are estimates for the variances and cross covariance of $G_{x}$ and $G_{y}$, averaged over the window $W$. The average gradient direction $\phi$ is given by:

$$
\phi=\frac{1}{2} \angle\left(G_{x x}-G_{y y}, 2 G_{x y}\right)
$$

where $\angle(x, y)$ is defined as:
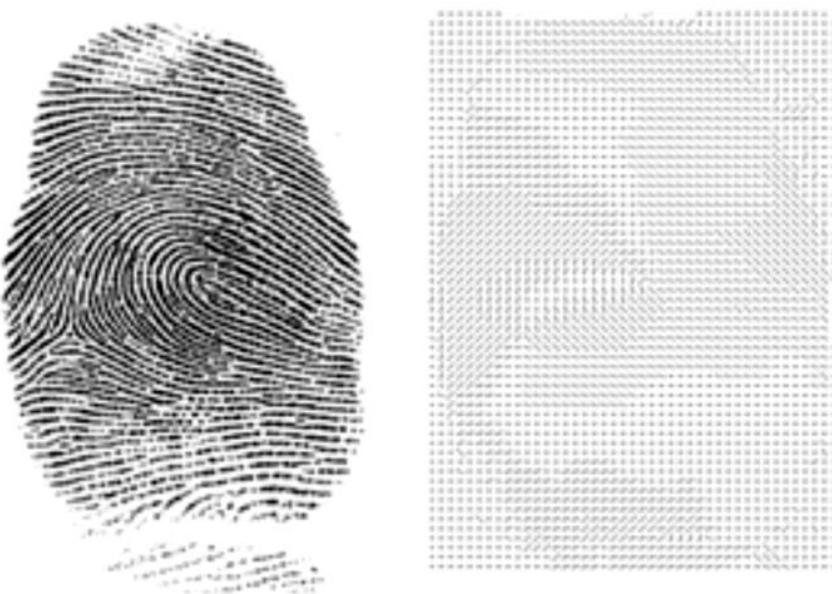

Fig.6. Fingerprint and Directional Field

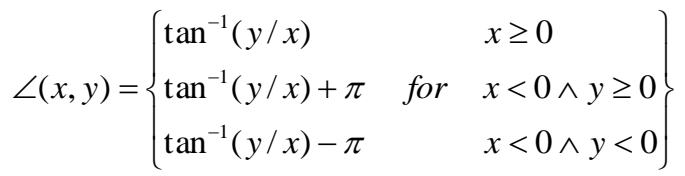

Directional field image obtained is shown in Fig. 6.

\section{B. Singularity Detection and Fingerprint Classification}

Singularities are the vertices of the fingerprint polygon. The most common method used forsingularity detection is by means of Poincaré index proposed by Kawogoe and Tojo, 1984[31].

Poincaré index is given by

$$
P P_{G C}(i, j)=\sum_{k=0 . .7} \operatorname{angle}\left(d_{k}, d_{(k+1) \bmod 8}\right)
$$

Where $G$ is the field associated with the fingerprint orientation image, $D . C$ is the closed path defined as an ordered sequence; $d$ is the directional field of individual blocks around region of interest (Table 1).

TABLE 1. POINCARÉ INDEX COMPUTATION SCHEME

\begin{tabular}{|c|c|c|}
\hline$d_{2}$ & $d_{3}$ & $d_{4}$ \\
\hline$d_{1}$ & {$[i, j]$} & $d_{5}$ \\
\hline$d_{0}$ & $d_{7}$ & $d_{6}$ \\
\hline
\end{tabular}

Poincaré index method cannot accurately detect the singular points for noisy or low quality fingerprints and for singular points in arch fingerprints and some of the tented arch fingerprints [32]. Coherence, which gives the strength of the orientation, measures how well all squared gradient vectors share the same orientation [26]. In this work, singularities are 


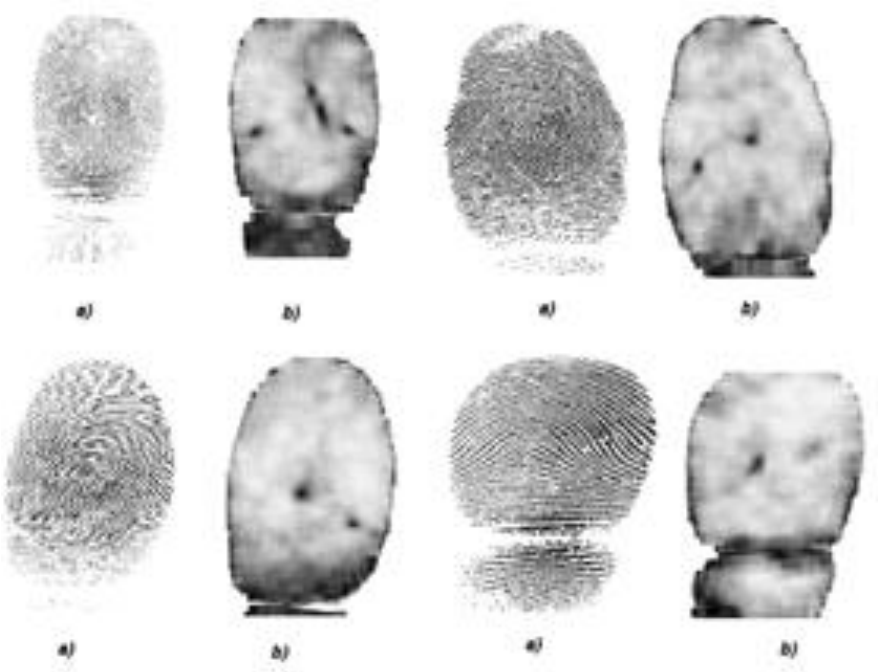

Fig.7. Fingerprints and Coherence Images

located using Coherence computed using squared gradients. The coherence of the squared gradients is given by:

$$
\operatorname{Coh}=\frac{\left|\sum_{W}\left(G_{s, x}, G_{s, y}\right)\right|}{\sum_{W}\left|\left(G_{s, x}, G_{s, y}\right)\right|}
$$

Fig.7 shows the coherence image formed with singularities clearly shown as dark spots. Depending on the relative position of the singularities, fingerprints are classified into seven types namely: Left Loop, Right Loop, Whorl without any Delta, Whorl with one Delta and whorl with two deltas, Arch and Tented Arch.

\section{Baseline detection and feature vector formation}

Majority of the fingerprint identification algorithms are based on minutiae and ridge features. In this work baseline is considered as the reference line for the fingerprint singularities. This line has to be detected accurately to form the fingerprint polygon. Hough Transform and other versions of Hough Transform based line identification are the most popular line identification technique used by image processing researchers [33], [34], [35]. Guru et al. [36] have proposed a PCA based method for line detection. In all the cases computational complexity is high. Also fingerprints are as such line patterns and hence identification of base line using Hough transform methods requires additional intelligence. In this work base line is detected using a correlation method as per the following steps [21]:

1. Since baseline falls in the lower portion of the fingerprint image, computation for line identification needs to be done only in the lower portion of fingerprint image and hence identification can be done below the centroid of the segmented fingerprint image.

2. Binary masks of sizes from $200 \times 50$ to $200 \times 3$ are defined. Mask of 200 X 50 is to detect most slanted base line (about $23^{\circ}$ ) and $200 \times 3$ is to detect a horizontal line. A portion of the mask of size $200 \mathrm{X}$ 50 is shown in Fig.8.

3. Find the normalized cross-correlation peak between each masks and the fingerprint regions using $S=M$ $\otimes F$; where $S$ is the correlation peak, $M$ and $F$ are mask and fingerprint regions respectively.

4. If $S \geq T$, a threshold peak, presence of the base line is identified and the baseline is drawn with reference to the mask direction.

\begin{tabular}{|l|l|l|l|l|l|l|l|l|l|l|l|l|l|l|l|l|l|l|l|l|l|l|}
\hline 0 & 0 & 0 & 0 & 0 & 0 & 0 & 0 & 0 & 0 & 0 & 0 & 0 & 0 & 0 & 0 & 0 & 0 & 0 & 0 & 0 & 0 & 0 \\
\hline 0 & 0 & 0 & 0 & 0 & 0 & 0 & 0 & 0 & 0 & 0 & 0 & 0 & 0 & 0 & 0 & 0 & 0 & 0 & 0 & 0 & 0 & 0 \\
\hline 1 & 1 & 1 & 1 & 1 & 0 & 0 & 0 & 0 & 0 & 0 & 0 & 0 & 0 & 0 & 0 & 0 & 0 & 0 & 0 & 0 & 0 & 0 \\
\hline 1 & 1 & 1 & 1 & 1 & 1 & 1 & 1 & 1 & 1 & 0 & 0 & 0 & 0 & 0 & 0 & 0 & 0 & 0 & 0 & 0 & 0 & 0 \\
\hline 1 & 1 & 1 & 1 & 1 & 1 & 1 & 1 & 1 & 1 & 1 & 1 & 1 & 1 & 1 & 0 & 0 & 0 & 0 & 0 & 0 & 0 & 0 \\
\hline 0 & 0 & 0 & 0 & 0 & 1 & 1 & 1 & 1 & 1 & 1 & 1 & 1 & 1 & 1 & 1 & 1 & 1 & 1 & 0 & 0 & 0 & 0 \\
\hline 0 & 0 & 0 & 0 & 0 & 0 & 0 & 0 & 0 & 0 & 1 & 1 & 1 & 1 & 1 & 1 & 1 & 1 & 1 & 1 & 1 & 1 & 1 \\
\hline 0 & 0 & 0 & 0 & 0 & 0 & 0 & 0 & 0 & 0 & 0 & 0 & 0 & 0 & 0 & 1 & 1 & 1 & 1 & 1 & 1 & 1 & 1 \\
\hline 0 & 0 & 0 & 0 & 0 & 0 & 0 & 0 & 0 & 0 & 0 & 0 & 0 & 0 & 0 & 0 & 0 & 0 & 0 & 1 & 1 & 1 & 1 \\
\hline 0 & 0 & 0 & 0 & 0 & 0 & 0 & 0 & 0 & 0 & 0 & 0 & 0 & 0 & 0 & 0 & 0 & 0 & 0 & 0 & 0 & 0 & 0 \\
\hline 0 & 0 & 0 & 0 & 0 & 0 & 0 & 0 & 0 & 0 & 0 & 0 & 0 & 0 & 0 & 0 & 0 & 0 & 0 & 0 & 0 & 0 & 0 \\
\hline
\end{tabular}

Fig.8. Baseline detection mask

Fig.9 shows the fingerprint base line detected for various image orientations. A polygon is formed from the finger baseline and the singularities and the angle and distance features are evaluated from this polygon. The ridge counts are obtained by counting the number of intensity minima between the desired singularities in the polygon.

\section{MULTIFINGER FEATURE SELECTION}

Let $X=\left\{x_{1}, x_{2}, \ldots x_{m}\right\}$ and $Y=\left\{y_{1}, y_{2}, \ldots y_{n}\right\}$ denotes the 16 element feature vectors $\left(x \in R^{m}\right)$ and $\left(y \in R^{n}\right)$ of the two fingers. The feature vectors of the combined fingers is defined as $Z=X \cup Y$. In this case feature normalisation is not required as the features are from same modality and are homogeneous. Thus a 32 element feature vector is formed.

\section{MATCH SCORE GENERATION}

Matching gives a numerical score which shows how much the input image (I) matches with the existing fingerprint template (T). Any fingerprint algorithm compares two given fingerprints and returns either a degree of similarity or a binary decision. The matching score which is a number in the range 0 to 1 is calculated as the ratio of the number of matched features to the total number of features. In this work we have formulated a simple matching score based on the Euclidean distance. The steps for computing the matching score are:
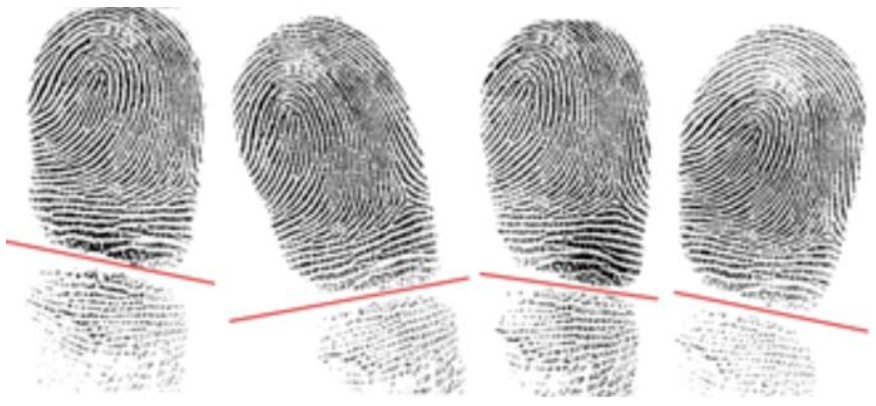

Fig. 9. Baseline detected

1. Find the Euclidean distances between the distance features and angles corresponding to input image $I$ and query template $T$. 
2. Compute matching score,

$$
M=\left\{\begin{array}{cc}
\frac{T h-\sum_{n=1}^{N}\left|I_{n}-F_{n}\right| * w_{n}}{T h} & \text { if } \sum_{n=1}^{N}\left|F_{n}-F_{n}\right| * w_{n}<T h \\
0 & \text { otherwise }
\end{array}\right.
$$

$T h$ is the Threshold value assigned, $w_{n}$ is the weight, $I_{n}$ and $F_{n}$ are the Input image and Template metrics, $N$ is the feature vector length.

3. If $M \leq T h$, fingerprint matches, where $0 \leq T h \leq 1$.

\section{IMPLEMENTATION OF THE ALGORITHM}

\section{A. Database used}

In our work consecutive fingerprint images (forefinger and middle finger) have been acquired using a fingerprint scanner with 500 dpi resolution and image size of 600 X 600 pixels. Fingerprint samples of about 300 persons were collected and features were extracted and stored.

\section{B. Implementation}

Individual fingerprints are segmented to detect the baseline and to generate the feature vectors. Baseline is captured clearly for individual fingerprints and the polygon is drawn for whorl, left loop, right loop and arch classes of the input images and the features from the polygon are evaluated. Classification of fingerprints is done for each fingerprint as per the classification scheme and the templates are formed and stored [Table 2].

\section{Results and Discussion}

About 300 fingerprint pairs were taken and the features were extracted and stored as template data. Another sample set of 300 fingerprint pairs of same persons were taken as test set. Match score has been calculated for each fingerprint in the test set with the template. Box plot, which is a statistical plot of the score distribution, is shown in fig.10. A genuine fingerprint is one which is supposed to match with the same fingerprint template in the template data. The genuine distribution shows a median of about 0.92 and the whiskers ranging between 0.72 to 1 . An imposter in one whose fingerprint does not matches with the template data. The imposter distribution shows a median of 0.56 with the whiskers ranging between 0 and 0.71 . Hence fixing a matching score threshold between 0.72 and 0.71 can identify all the fingerprints with $100 \%$ accuracy. The Receiver Operator Characteristics (ROC) graph is shown in fig.11. The False Acceptance Rate(FAR) is a measure of how many imposter users are falsely accepted into the system as "genuine" users is plotted by varying the threshold from 0 to 1. The False Rejection Rate (FRR) is a measure of how many genuine users are falsely rejected by the system as "imposters" is also calculated for various thresholds and is plotted. The Equal Error Rate (EER) is defined as the condition at which FAR=FRR as in Figure 11, is approximately equal to zero at a threshold of $T h=0.715$. For this threshold, fingerprints are identified with $100 \%$ accuracy. Fig. 12 shows the ROC correspond to single finger based identification hich shows a high threshold can only identify the fingerprint with $100 \%$ accuracy.

TABLE 2. FINGERPRINT TEMPLATE

\begin{tabular}{|c|c|c|c|c|c|c|c|c|c|c|c|c|c|c|c|}
\hline$d_{c c}$ & $d_{c b}$ & $d_{c d r}$ & $d_{d b r}$ & $d_{b b}$ & $d_{d b l}$ & $d_{c d l}$ & $\theta_{C}$ & $\theta_{D R}$ & $\theta_{D L}$ & $\theta_{C C}$ & $A$ & $T$ & $r_{c d}$ & $r_{c b}$ & $r_{d b}$ \\
\hline 0.00 & 234.47 & 0.00 & 0.00 & 110.74 & 181.77 & 122.70 & 60.13 & 0.00 & 119.88 & 0.00 & 23049.00 & 2 & 10.67 & 18.67 & 15.33 \\
\hline 0.00 & 219.56 & 0.00 & 0.00 & 86.41 & 151.14 & 110.49 & 44.77 & 0.00 & 135.18 & 0.00 & 16055.00 & 2 & 11.00 & 21.00 & 13.33 \\
\hline 0.00 & 258.32 & 0.00 & 0.00 & 141.61 & 144.20 & 179.13 & 50.25 & 0.00 & 129.55 & 0.00 & 27670.75 & 2 & 17.50 & 16.75 & 10.50 \\
\hline 0.00 & 256.01 & 181.95 & 120.13 & 121.06 & 0.00 & 0.00 & 41.82 & 138.10 & 0.00 & 0.00 & 22805.50 & 1 & 13.67 & 15.17 & 8.33 \\
\hline 0.00 & 252.18 & 0.00 & 0.00 & 109.93 & 142.25 & 155.49 & 44.95 & 0.00 & 135.15 & 0.00 & 21605.67 & 2 & 13.33 & 21.50 & 14.50 \\
\hline 0.00 & 237.91 & 0.00 & 0.00 & 12.02 & 187.32 & 52.30 & 13.33 & 0.00 & 166.66 & 0.00 & 2567.25 & 2 & 3.00 & 15.50 & 14.75 \\
\hline 0.00 & 253.24 & 0.00 & 0.00 & 44.01 & 218.95 & 56.31 & 52.07 & 0.00 & 127.82 & 0.00 & 10451.00 & 2 & 7.00 & 20.00 & 16.50 \\
\hline 0.00 & 317.00 & 0.00 & 0.00 & 152.00 & 124.00 & 245.67 & 17.12 & 0.00 & 162.88 & 0.00 & 33516.00 & 2 & 17.00 & 18.00 & 6.00 \\
\hline 0.00 & 250.12 & 0.00 & 0.00 & 101.24 & 174.45 & 126.45 & 47.78 & 0.00 & 132.20 & 0.00 & 21503.25 & 2 & 11.00 & 20.75 & 14.00 \\
\hline 0.00 & 231.18 & 0.00 & 0.00 & 43.44 & 172.38 & 73.14 & 36.61 & 0.00 & 143.34 & 0.00 & 8782.83 & 2 & 5.00 & 21.50 & 16.00 \\
\hline 0.00 & 281.00 & 0.00 & 0.00 & 125.00 & 105.00 & 193.72 & 21.79 & 0.00 & 158.21 & 0.00 & 25875.00 & 2 & 16.00 & 21.50 & 9.00 \\
\hline 0.00 & 271.20 & 0.00 & 0.00 & 124.81 & 103.71 & 197.70 & 29.88 & 0.00 & 150.28 & 0.00 & 24235.75 & 2 & 16.00 & 17.50 & 9.25 \\
\hline 0.00 & 297.74 & 182.66 & 129.60 & 71.56 & 0.00 & 0.00 & 23.05 & 156.96 & 0.00 & 0.00 & 15289.25 & 1 & 15.00 & 20.50 & 8.00 \\
\hline 0.00 & 230.95 & 158.13 & 121.03 & 113.58 & 0.00 & 0.00 & 45.92 & 134.14 & 0.00 & 0.00 & 19952.33 & 1 & 13.67 & 17.00 & 9.33 \\
\hline 0.00 & 249.02 & 102.70 & 171.14 & 66.85 & 0.00 & 0.00 & 37.23 & 142.76 & 0.00 & 0.00 & 14043.33 & 1 & 8.33 & 18.17 & 12.17 \\
\hline 0.00 & 233.79 & 91.84 & 184.95 & 77.24 & 0.00 & 0.00 & 55.65 & 124.32 & 0.00 & 0.00 & 16181.00 & 1 & 10.33 & 23.33 & 14.00 \\
\hline 163.77 & 129.25 & 221.05 & 114.21 & 101.18 & 0.00 & 0.00 & 168.02 & 142.36 & 0.00 & 154.31 & 20160.00 & 5 & 12.00 & 14.00 & 16.00 \\
\hline
\end{tabular}




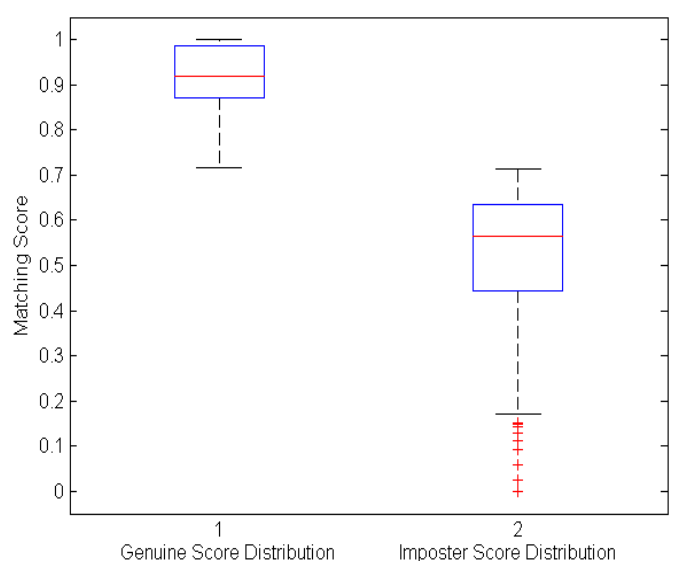

Fig.10. Score Distribution - Multifinger

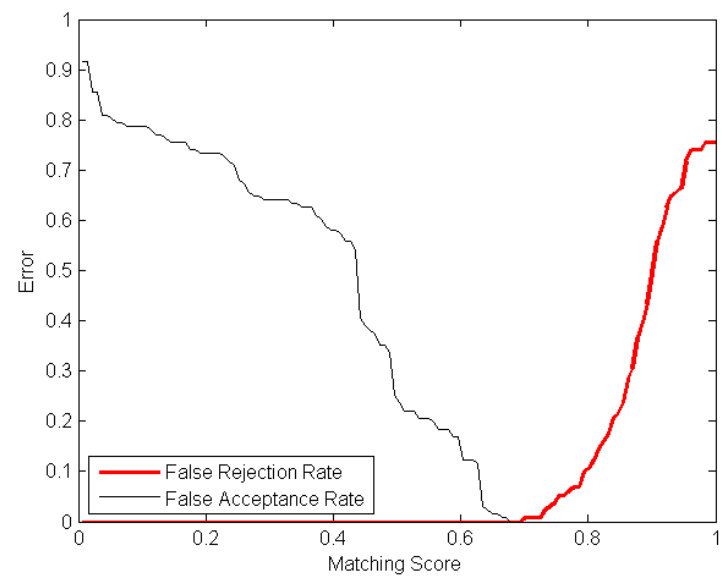

Fig.11. FAR-FRR Graph- Multifinger

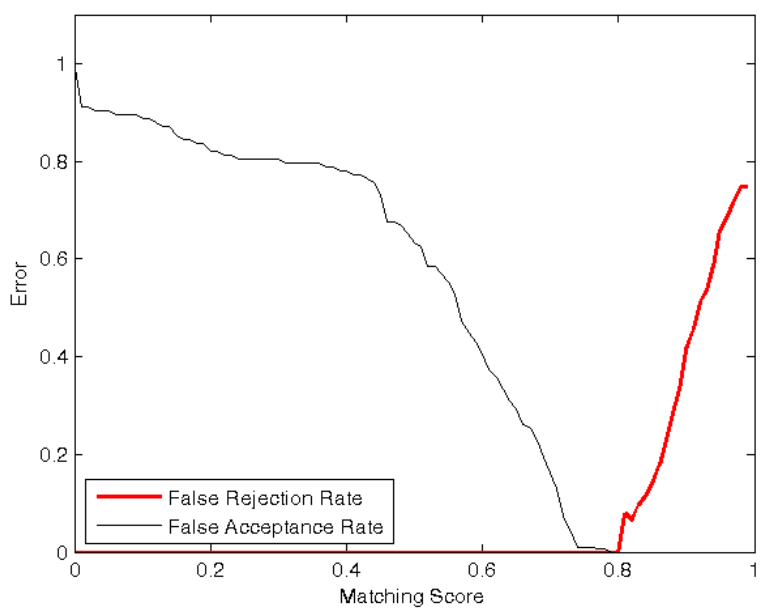

Fig.12 FAR-FRR Graph- Single finger based

\section{CONCLUSION}

Multifinger fusion based fingerprint identification is presented here. Singularities of consecutive individual fingerprints were found out using coherence computed via directional field strength. Fingerprint polygon was constructed for each fingerprints with the baseline detected and feature vectors were concatenated to form a 32 element vector. A distance based matching score was formulated and was tested with an accuracy of $100 \%$ detection for a database of 300 candidates.

\section{REFERENCES}

[1] David Maltoni, Dario Maio, Anil K Jain, Salil Prabhakar, Handbook of fingerprint recognition, $2^{\text {nd }}$ Ed. Springer, 2005.

[2] A.K. Jain, L. Hong and R. Bolle, "On-line fngerprint verification", IEEE Trans. Pattern Anal. Mach Intell. 194 (1997), pp. 302-314.

[3] A.K. Jain, S. Prabhakar and S. Pankanti, "Filterbank-based fingerprint matching", IEEE Trans. Image Process. 95 (2000), pp. 846-859.

[4] Nalini Ratha, Ruud Bolle Editors, Automatic Fingerprint Recognition Systems, Springer, 2003.

[5] R. Cappelli and D. Maio, "The state of the Art in Fingerprint Classification", Automatic Fingerprint Recognition Systems, N. Ratha and R. Bolle, eds., Springer, 2004.

[6] R. Cappelli, D. Maio and D. Maltoni, "Indexing Fingerprint Databases for Efficient 1: N Matching", Proc. Sixth International Conf. Control, Automation, Robotics and Vision, Dec. 2000.

[7] Raffaele Cappelli and Davide Maltoni, "On the Spatial Distribution of Fingerprint Singularities", IEEE Transactions on Pattern Analysis and Machine Intelligence, Vol 31, No. 4, April 2009 pp- 742-748.

[8] A Ross, A Jain, "Information fusion in biometrics", Pattern Recogn. Lett. 24 (2003)2115-2125

[9] Jain A K, Nandakumar K, and Ross A, "Score Normalization in Multimodal Biometric Systems", Pattern Recognition, 38(12), 22702285

[10] Arun A Ross, Karthik Nandakumar, Anil K Jain, Handbook of Multibiometrics, Springer, 2006.

[11] Biometrics in the Age of Heightened Security and Privacy. Available at http : // www.itl.nist.gov/div895/isis/bc/bc2001/

EDIT FINAL DR.ATTICK.pdf

[12] Most M, "Battle of Biometrics", Digital ID World Magazine, 2003, Pages 16-18.

[13] D L Hall, J Llinas, "Multisensor data fusion", D L Hall, J Llinas (Eds.), Handbook of Multisensor Data Fusion, CRC Press, 2001, pp 1-10.

[14] C. Sanderson, K.K. Paliwal, "Identity Verification Using Speech and Face Information", Digital Signal Processing, Vol. 14, No. 5, 2004, pp. 449-480.

[15] R Frischholz and U Dieckmann, Biod: "A multimodal biometric identification system”, IEEE Computer, 33(2), pp 64-68, 2000.

[16] L Hong and A K Jain, "Integrating faces and fingerprints for personal identification", IEEE Trans. on Pattern Analysis and Machine Intelligence 20(12), p 1295-1307, 1998.

[17] E Bigun, J Bigun, B Due and S Fischer, "Expert conciliation for multi modal person authentication by Bayesian statistics", Proc. of Int'l Conf. on Audio and Video based Person Authentication, pp 311-318, 1997.

[18] Y.Wang, T Tan and A. K. Jain, "Combining face and iris biometrics for identitiy verification", Proc. of Int'l Conf. on Audio and Video based Person Authentication, pp 805-813, 2003.

[19] A Kumar and D Zhang, "Integrating palmprint with face for user authentication", Workshop on Multi Modal User Authentication (MMUA), pp 107-112, 2003. 
[20] Arun Ross and Rohin Govindarajan, "Feature Level Fusion Using Hand and Face Biometrics", Proc. of SPIE Conference on Biometric Technology for Human Identification II, Vol 5779, pp 196-204, 2005.

[21] N Praveen and Tessamma Thomas, 'Singularity Based Fingerprint identification", International Journal of Research and Reviews in Computer Science (IJRRCS) Vol. 2, No. 5, pp 1055-1059, October 2011.

[22] Asker M Bazen and Sabih H. Gerez, "Systematic Methods for the Computation of the Directional Fields and Singular Points of Fingerprints", IEEE Transactions on Pattern Analysis and Machine Intelligence, Vol. 24, No. 7, July, 2002 pp 905-919.

[23] G. A. Dretsand G. G. Liljenstr'Om, "Fingerprint Sub classification: A Neural Network Fingerprint Classification", Intelligent Biometric Techniques in Fingerprint and Face Recognition, L C. Jain, U. Halici, I. Hayashi,, S. B. Lee, and S. Tsutsui, eds., pp. 109-134, Boca Raton, Fla.: CRC Press, 1999.

[24] C. L. Wilson, G. T. Candela, and C. I. Watson, "Neural Network Fingerprint Classification", J. Artificial Neural Networks, Vol. 1, No. 2, pp. 203-228, 1994.

[25] L'O. Gorman and J. V. Nicherson, "An Approach to Fingerprint Filter Design”, Pattern Recognition, Vol. 22, No. 1, pp.-29-38, 1989.

[26] M. Kass and A. Witkin, "Analyzing Oriented Patterns", Computer Vision, Graphics, and Image Processing, Vol. 37, No. 3, pp.-362-385, March 1987.

[27] A. K Jain, L. Hong, S. Pankanti, and R. Bolle, 'An Identity Authentication System Using Fingerprints”, Proc. IEEE, Vol. 85, no. 9, pp1365-1388, Sept. 1997.

[28] A. R. Rao and R. C. Jain,Computerized, "Flow Field Analysis: Oriented Texture Fields", IEEE Transactions on Pattern Analysis and Machine Intelligence, Vol. 14, no. 7,, pp. 693- 709, July, 1992.

[29] N. Ratha, S. Chen, and A. Jain, "Adaptive Flow Orientation Based Feature Extraction in Fingerprint Images", Pattern Recognition, vol. 28, pp. 1657-1672, Nov. 1995.

[30] P. Perona, "Orientation Diffusions", IEEE Trans. Image Processing, vol.7, no.3, pp.457-467, Mar.1998.
[31] Masahiro Kawagoe and Akio Tojo, "Fingerprint pattern classification", Pattern Recognition, Vol. 17, no.3, pp 295-303,1984 .

[32] CHENG Xin-Ming, XU Dong-Cheng, XU Cheng, "A New Algorithm for Detecting Singular Points in Fingerprint Images", Third International Conference on Genetic and Evolutionary Computing, 2009.

[33] Atiquzzaman, M., 1992. "Multiresolution Hough transform-an efficient method of detecting patterns in images", IEEE Trans. Pattern Anal.Machine Intell. 14 (11), 1090-1095.

[34] Duda, R.O., Hart, P.E., 1972. "Use of Hough transformation to detect lines and curves in pictures", Commun. ACM 15 (1), 11-15.

[35] Hough P.V.C., 1962. "Method and means for recognizing complex patterns", U.S. Patent No. 3069654.

[36] D. S. Guru B, H. Shekar, P. Nagabhushan, "A simple and robust line detection algorithm based on small Eigen value analysis", Pattern Recognition. 25, pp. 1-13, 2003.

\section{AUTHORS PROFILE}

Praveen $\mathbf{N}$ received his MSc (Electronics) from Department of Electronics, Cochin University of Science and Technology, Kochi, 682022. He is working as Associate Professor of Electronics in the Department of Electronics, N S S College, Rajakumari, Idukki Dt., Kerala. He has 15 years of teaching experience and five years of research experience. At present he is doing $\mathrm{PhD}$ in Department of Electronics, Cochin University of Science and Technology. His research areas of interest include digital signal / image processing, biometrics, computer vision etc.

Dr.Tessamma Thomas received her M.Tech and Ph.D from Cochin University of Science and Technology, Cochin-22, India. At present she is working as Professor in the Department of Electronics, Cochin University of Science and Technology. She has to her credit more than 90 research papers, in various research fields, published in International and National journals and conferences. Her areas of interest include digital signal / image processing, bio medical image processing, super resolution, content based image retrieval, genomic signal processing etc. 